\title{
Near-field optical investigation of three-dimensional photonic crystals
}

\author{
E. Flück, ${ }^{1}$ N. F. van Hulst, ${ }^{1}$ W. L. Vos, ${ }^{2}$ and L. Kuipers ${ }^{1, *}$ \\ ${ }^{1}$ Applied Optics Group, Department of Science and Technology and MESA ${ }^{+}$Research Institute, University of Twente, P.O. Box 217, \\ 7500 AE Enschede, The Netherlands \\ ${ }^{2}$ Complex Photonic Systems, Department of Science and Technology and MESA ${ }^{+}$Research Institute, University of Twente,
} P.O. Box 217, 7500 AE Enschede, The Netherlands

(Received 11 February 2003; published 3 July 2003)

\begin{abstract}
We show that the coupling of light from an external pointlike light source into a three-dimensional photonic crystal depends on the relative launching position with respect to the crystal lattice as well as on the frequency of light. The results are obtained with a near-field technique which is used to acquire optical information beyond the diffraction limit and to access optical details within the unit cell of the crystal. The experiments are performed at frequencies near the second-order L-gap. As a result, the changes in the shape of the near-field pattern are explained by the photonic properties of the crystal.
\end{abstract}

DOI: 10.1103/PhysRevE.68.015601

PACS number(s): 42.70.-a, 42.25.-p

\section{INTRODUCTION}

Photonic crystals are three-dimensional (3D) periodic dielectric composites, with periods of the order of the wavelength of light $[1,2]$. These crystals are attracting a great deal of interest as they offer a huge potential for controlling both the spontaneous emission of embedded light sources and the propagation of light itself. Photonic crystals are rarely considered as the complex optical structures they are; their complete description should take into account not only the farfield but also the near-field properties [3]. So far, two main approaches have been utilized to probe the optical properties of photonic crystals. The first and by far the most extensively used has been transmission or reflection experiments in which both source and detector are in the far field, see, e.g., Refs. [4,5]. These far-field methods have been successfully used to determine stopgaps, and mean free paths, and to elucidate the propagation of ultrafast pulses. Second, far-field measurements of spontaneous emission rates of sources embedded inside the crystals probe the so-called local density of states (LDOS) $[1,2]$. The LDOS contains both far- and nearfield components, and gives rise to modified quantum electrodynamics including inhibited or enhanced emission [6]. In both approaches, the optical properties are spatially averaged by virtue of the detector being in the far-field. Even if focused beams are used $[7,8]$, diffraction still limits the obtainable spatial resolution.

In this paper, we present a method to probe the near-field properties of $3 \mathrm{D}$ photonic crystals. A subwavelength light source near the surface of the crystal launches light into the crystal, i.e., artificial opals made of colloidal spheres. By scanning the position of the light source relative to the crystal surface, we measure the amount of light that reaches the other side of the crystal as a function of the launch position. The measurements are performed for different frequencies. With a resolution superior to the above mentioned techniques, we observe spatial variations in the amount of light

\footnotetext{
*Present address: FOM-Institute for Atomic and Molecular Physics, Kruislaan 407, 1098 SJ Amsterdam, The Netherlands.
}

launched into the crystals on length scales smaller than a unit cell. It is established that the observed near-field patterns are independent of the experimental conditions such as the nearfield probe geometry and the height feedback. The measurements have been reproduced with four different near-field probes. We find that more light is launched into the photonic crystal if the probe is located directly above the spheres. The intensity maxima shift and change their shape for different frequencies of light situated near the secondorder $L$-gap of the artificial opal. We therefore show that the coupling of light from an external source into a 3D photonic crystal depends on both position and frequency.

\section{EXPERIMENT}

The instrument used is a near-field scanning optical microscope (NSOM) in illumination mode [9]. The near-field optical fiber probes with subwavelength apertures are fabricated from single-mode optical fibers, which are locally heated and subsequently pulled to achieve a sharp taper region with a small end face $(\sim 80 \mathrm{~nm})$. Next, the fiber probes or tips are coated with an aluminum layer of $\sim 100 \mathrm{~nm}$ thickness. To produce a circular aperture in the coating at the end facet of the tip, the coating process is performed under an angle. The apertures are cleaned up or enlarged to every desired dimension through side-on milling with a focused ion beam [10]. The fabrication process of our fiber probes is highly reproducible, and produces probes with a throughput of typically $10^{-5}$ and better. Single molecule investigations at different wavelength have proven that the near-field probe emission is independent of the wavelength used [11]. Figure 1 shows a schematic drawing of the experimental setup. To perform a measurement, the fiber probe is $x-y$ raster scanned at a constant probe-surface separation of $z \sim 10 \mathrm{~nm}$, using a shear-force feedback mechanism [12]. As a result, the topographical information of the samples under investigation is simultaneously measured with the optical information. For all measurements presented here, the same tip was used. The measurements have been reproduced with four tips of similar aperture sizes. Directly beneath the crystal, a photodiode is positioned to collect the transmitted light. With our detection 


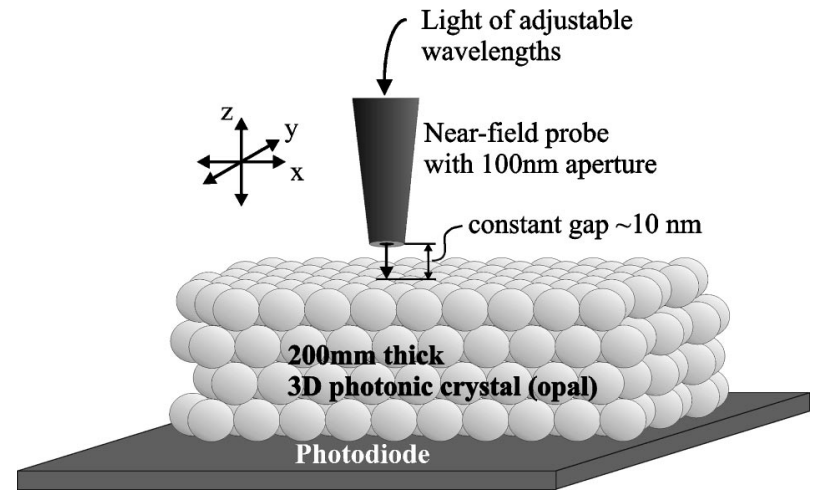

FIG. 1. Schematic drawing of the near-field setup. From a point source (near-field aperture probe), light is launched to the 3D photonic crystal. A photodiode, mounted underneath the crystal, detects all the light reaching the other side of the crystal. By $x-y$ scanning of the fiber probe relative to the crystalline $\langle 111\rangle$ surface, the light transfer is studied as a function of launching position.

method all light coming from the bottom crystal facet is detected. This is beneficial, since coupling effects from the out-coupling facet are avoided. In contrast to this, a conventional NSOM setup uses a high numerical aperture objective to detect only the light exiting in a small area of the out-coupling facet $[9,13]$.

The near-field investigations were performed on selfassembled polystyrene opals with a sphere radius $r=241$ $\pm 3 \mathrm{~nm}$ [14]. The crystals show a long-range fcc order and present the $\langle 111\rangle$ interface to the near-field fiber probe. The first-order $L$-gap is at $1140 \mathrm{~nm}$, whereas the second-order $L$ gap is near $600 \mathrm{~nm}$. Both $L$-gaps were probed experimentally by reflectivity measurements, where the measured peak widths were $8 \%$ and $3 \%$, respectively. We used three different wavelengths of light, which are situated near the secondorder $L$-gap region.

\section{RESULTS AND DISCUSSION}

In Fig. 2, the transfer of light of $\lambda_{0}=647 \mathrm{~nm}$ launched from the near-field fiber probe is shown. As a first step, we have carried out optical measurements at different planes of constant height above the surface [15]. Figure 2(a) shows the topographical information obtained simultaneously with the near-field pattern shown in Fig. 2(b). Topographical corrugations of up to $85 \mathrm{~nm}$ are obtained. The high topographical resolution is a result of a small $\mathrm{Al}$ grain located at the end face of the fiber probe. The grainy structure of the tip can also be seen due to tip convolution in Fig. 2(a). The detected optical signals for four different tip heights above the crystal are shown in Fig. 2(b) with $z \sim 10 \pm 5 \mathrm{~nm}$ (in shear-force feedback), Fig. 2(c) with $z=84 \pm 30 \mathrm{~nm}$, Fig. 2(d) $z=446$ $\pm 30 \mathrm{~nm}$, and Fig. 2(e) with $z=723 \pm 16 \mathrm{~nm}$. In all four measurements, a hexagonal pattern of bright intense spots is clearly visible, which remains even for large tip to sample separation, and no influence in the near-field pattern in Fig. 2(b) due to the height feedback is observed. We therefore exclude the topographical artifacts, such as those charted by, for example, Hecht et al. [16]. The periodicity of the optical
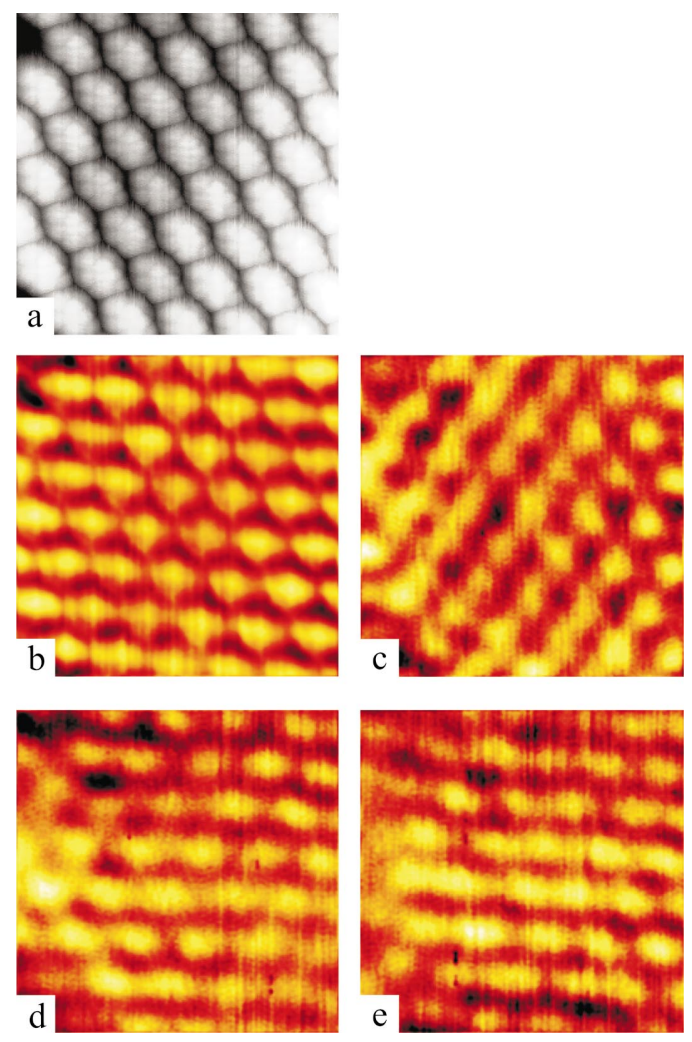

FIG. 2. (Color) Near-field measurements performed for different tip to sample separations. (a) Topographical image where the hexagonal arrangement of the polystyrene spheres in the $\langle 111\rangle$ surface of the crystal is visible. The apparent shape of the spheres is dominated by so-called tip convolution effects. (b) Detected intensity versus $x-y$ position, obtained simultaneously with image (a) at $z$ $\sim 10 \mathrm{~nm}$. (c)-(e) Detected spatial intensity in planes parallel to the $\langle 111\rangle$ surface at $z=84 \mathrm{~nm}, z=446 \mathrm{~nm}$, and $z=723 \mathrm{~nm}$, respectively. The optical patterns show local information about the light transfer with subwavelength resolution, and the pattern persists for different tip to sample separations. Image sizes: $2.71 \times 3.02 \mu \mathrm{m}^{2}$.

pattern corresponds to the arrangement of the polystyrene spheres, which is $2 r=482 \mathrm{~nm}$. Thus in all images subwavelength resolution is observed. This indicates that the nearfield properties can be investigated directly with our method.

A more detailed analysis of the measurements in Fig. 2 reveals that the optical modulation $M=\left(I_{\max }-I_{\min }\right) /\left(I_{\max }\right.$ $\left.+I_{\text {min }}\right)$ decreases as a function of tip to sample separation from $7 \%$ in Fig. 2(b) to $4 \%$ in Fig. 2(e). Furthermore, the intensity of the transmitted light through the crystals drops by $13 \%$ with increasing tip to sample separation from $z$ $\sim 10 \mathrm{~nm}$ to $z=723 \mathrm{~nm}$. This increase at smaller distances indicates that more light is launched into the crystal when the tip is in the near-field regime than when the tip is in the far-field regime. Since our near-field probe can be seen as a pointlike light source, wave vectors over an angular range of $2 \pi$ solid angle are available. The increase in throughput, when working in the near-field regime, strongly indicates that wave vectors beyond the critical angle are coupled to the crystal.

As a next step, light of different wavelengths is launched into the crystal with the same probe. The wavelengths used 

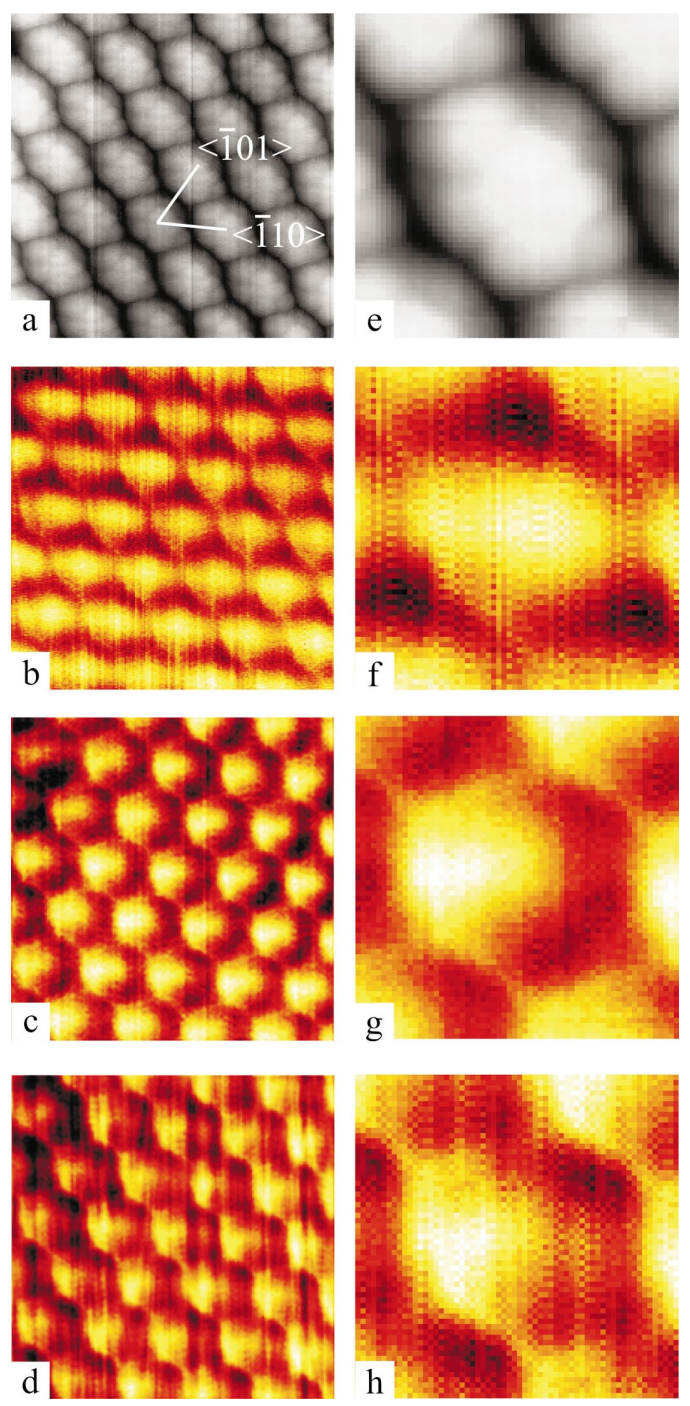

FIG. 3. (Color) Position-dependent ( $x-y$ plane) transfer of light from tip to photonic crystal obtained for different frequencies of light. (a) Topographical information of the $\langle 111\rangle$ crystalline surface of the polystyrene crystal. (b) -(d) Near-field optical patterns at $\lambda_{0}$ $=647 \mathrm{~nm}, 568 \mathrm{~nm}$, and $514 \mathrm{~nm}$, respectively. Image sizes: 2.28 $\times 2.53 \mu \mathrm{m}^{2}$. (e)-(h) show the topography and the optical signals of a close-up area around one polystyrene sphere in more detail. The images are built up from an average unit cell to sharpen the details of the original measurements. Image sizes: $0.65 \times 0.72 \mu \mathrm{m}^{2}$.

$\left(\lambda_{0}=647 \mathrm{~nm}, 568 \mathrm{~nm}\right.$, and $514 \mathrm{~nm}$ ) are situated below and in the second-order $L$-gap region of the crystal. Figure 3(a) shows the topography of the polystyrene crystal obtained simultaneously with the optical information shown in Fig. 3(d). The topographical information for the other optical measurements is identical to Fig. 3(a). Figures 3(b)-3(d) show the obtained near-field pattern for $\lambda_{0}=647 \mathrm{~nm}, 568$ $\mathrm{nm}$, and $514 \mathrm{~nm}$, respectively. In all three measurements, the hexagonal structure of the sphere arrangement with period $2 r=482 \mathrm{~nm}$ is clearly present. For all three different measurements, more light is detected when the tip is positioned on the top of a sphere. Less light is detected when the fibre probe is between the spheres. The variation of intensity as a

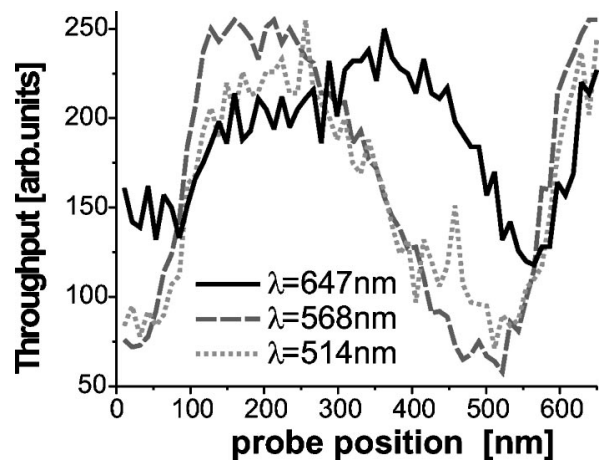

FIG. 4. Line traces along the crystalline $\langle\overline{1} 10\rangle$ direction from Figs. 3(f)-3(h). The shape of the optical pattern changes as a function of frequency. This is elucidated by determining the width $w$ of the bright spots, which is $w=370 \mathrm{~nm}$ at $\lambda_{0}=647 \mathrm{~nm}$, and $w$ $=280 \mathrm{~nm}$ at $\lambda_{0}=568 \mathrm{~nm}$ and at $\lambda_{0}=514 \mathrm{~nm}$ for the $\langle\overline{1} 10\rangle$ crystalline direction.

function of position is in the order of $\pm 6 \%$ of the average intensity. This position-dependent transfer of the light has to our knowledge never been observed before. The images obtained contain all the information about launching light into the thick 3D photonic crystal, the propagation of this light through, and its exit from the crystal.

We find an obvious change in the optical image for different wavelengths of light. To elucidate the changes in more detail, we summed up all the unit cells found in the measurements in Figs. 3(b)-3(d). From the resulting average unit cell, an area around one sphere is rebuilt, which gives a more pronounced image of the detailed information. Figure 3(e) shows the topography of the rebuilt area around one sphere. The slight apparent distortion of the unit cell is ascribed to imperfections of the tip. Figures 3(f)-3(h) show the corresponding optical fields for the three different wavelengths. It is clearly seen that the position of the bright spot shifts from the center of the sphere for $\lambda_{0}=647 \mathrm{~nm}$ to one edge of the sphere for $\lambda_{0}=514 \mathrm{~nm}$. Interestingly, the threefold symmetry around the $\langle 111\rangle$ crystalline direction is broken in Figs. $3(\mathrm{~g})$ and $3(\mathrm{~h})$.

\begin{tabular}{ccc} 
Crystalline direction & $\begin{array}{c}\text { Green-red } \\
\Delta(\mathrm{nm})\end{array}$ & $\begin{array}{c}\text { Yellow-red } \\
\Delta(\mathrm{nm})\end{array}$ \\
\hline$\langle\overline{1} 10\rangle$ & $82 \pm 6$ & $72 \pm 6$ \\
$\langle\overline{1} 01\rangle$ & $24 \pm 9$ & $23 \pm 9$
\end{tabular}

To investigate the shift of the bright intensity spots, we have performed cross correlations between the different optical pattern of Figs. 3(b)-3(d). As a result, the shift of the bright spots can be determined for the different crystalline directions. In the table below, the obtained shifts along the crystalline $\langle\overline{1} 10\rangle$ and $\langle\overline{1} 01\rangle$ directions are given. We observe that the shift between green and red $\left(\lambda_{0}=514 \mathrm{~nm}\right.$ and 647 $\mathrm{nm})$ along the $\langle\overline{1} 10\rangle$ direction is larger than the shift observed between yellow and red $\left(\lambda_{0}=568\right.$ and $\left.647 \mathrm{~nm}\right)$ along the same crystalline axis. Along the $\langle\overline{1} 01\rangle$ direction, the shifts observed are comparable for green-red and yellow-red. 
As can be seen in Figs. 3(f)-3(h), not only the positions of the bright spots shift, but also their shape changes as a function of wavelength. Figure 4 depicts a line trace taken along the $\langle\overline{1} 10\rangle$ direction and through the center of the sphere from the rebuilt areas of Figs. 3(f)-3(h). The size of the bright intensity spots becomes smaller for shorter wavelengths. We measure the width $w$ of the bright spot to be $w=370 \mathrm{~nm}$ at $\lambda_{0}=647 \mathrm{~nm}$, whereas the width of the spots at $\lambda_{0}=568 \mathrm{~nm}$ and $\lambda_{0}=514 \mathrm{~nm}$ is $w=280 \mathrm{~nm}$.

To interpret the different optical patterns shown in Fig. 3, we suggest a tentative explanation. The origin of the complex and asymmetric optical patterns should be sought in the influence of the second-order stopgap region. We recall that the wavelengths used in our experiment are near the secondorder Bragg reflection region near $600 \mathrm{~nm}$. The frequency corresponding to $\lambda_{0}=647 \mathrm{~nm}$ is situated below the stopgap. For these wavelength, propagating Bloch modes can be excited. These Bloch modes require the symmetry of the crystal, which is clearly observed in our measurements. For $\lambda_{0}$ $=514 \mathrm{~nm}$ and $\lambda_{0}=568 \mathrm{~nm}$, the corresponding frequencies are situated inside the stopgap region. As a result, no propagating Bloch modes exist. For frequencies in a stopgap, the intensity distribution does not have the symmetry of the underlying crystals, and the distribution shifts through the unit cell. This hypothesis is based on reasoning from dynamical diffraction theory for $\mathrm{x}$ rays [17].

\section{CONCLUSIONS}

In conclusion, we have presented the local investigation of the coupling mechanism of light coming from a pointlike light source to a 3D photonic crystal. Our NSOM setup allows the determination of the position-dependent transfer of light with nanometer accuracy, which is less than the period of the photonic crystal. It is impossible to obtain this information with far-field methods. The observed near-field pattern persists for larger tip to sample separations. The observed optical patterns reveal that the light is launched more efficiently on top of the spheres rather than between the spheres. We observe a shift of the high intensity spots as a function of frequency. Moreover, the shape of these spots changes as a function of frequency. The fact that the secondorder Bragg reflection $(\Gamma-L)$ is situated near $600 \mathrm{~nm}$ explains the similarity of the optical images at $\lambda_{0}=514 \mathrm{~nm}$ and $\lambda_{0}=568 \mathrm{~nm}$, as well as the difference with the measurements performed at $\lambda_{0}=647 \mathrm{~nm}$. More extensive experimental work is currently in progress. Work on the theoretical understanding of near-field coupling to structures is still in its infancy. The work of Carminati and Sáenz [18] and Colas des Francs et al. [19] as well as finite difference time domain (FDTD) simulations [20] may contribute to the understanding of the new insight about the light transfer, which is gained from the measurements presented in this paper.

The authors thank Lydia Bechger for the fabrication of the samples and Jeroen Korterik for technical support on the NSOM setup. This work is part of the research program of the "Stichting voor Fundamenteel Onderzoek der Materie (FOM)," which is financially supported by the "Nederlandse Organisatie voor Wetenschappelijk Onderzoek (NWO)." The research is also part of the strategic research orientation "Advanced Photonic Structures" of the MESA ${ }^{+}$Research Institute.
[1] Photonic Crystals and Light Localization in the 21st Century, Vol. 563 of Nato Advanced Studies Institute, Series C: Mathematical and Physical Sciences, edited by C. M. Soukoulis (Kluwer, Dordrecht, 2001).

[2] K. Sakoda, Optical Properties of Photonic Crystals, Springer Series in Optical Sciences, Vol. 80 (Springer, New York, 2001).

[3] J.D. Jackson, Classical Electrodynamics (Wiley, New York, 1998).

[4] P. Mach, P. Wiltzius, M. Megens, D.A. Weitz, K.H. Lin, T.C. Lubensky, and A.G. Yodh, Phys. Rev. E 65, 031720 (2002).

[5] A.F. Koenderink, P.M. Johnson, J.F. Galisteo López, and W.L. Vos, C. R. Physique 3, 67 (2002).

[6] K. Busch, N. Vats, S. John, and B.C. Sanders, Phys. Rev. E 62, 4251 (2000).

[7] Y.A. Vlasov, M. Deutsch, and D.J. Norris, Appl. Phys. Lett. 76, 1627 (2000).

[8] J.F. Galisteo López and W.L. Vos, Phys. Rev. E 66, 036616 (2002).

[9] A.G.T. Ruiter, J.-A. Veerman, M.F. Garcia-Parajo, and N.F. van Hulst, J. Phys. Chem. A 101, 7318 (1997).
[10] J.-A. Veerman, A.M. Otter, L. Kuipers, and N.F. van Hulst, Appl. Phys. Lett. 72, 3115 (1998).

[11] J.-A. Veerman, M.F. Garcia-Parajo, L. Kuipers, and N.F. van Hulst, J. Microsc. 194, 477 (1999).

[12] K. Karrai and R.D. Grober, Appl. Phys. Lett. 66, 1842 (1995).

[13] E.B. McDaniel, J.W.P. Hsu, L.S. Goldner, R.J. Tonucci, E.L. Shirley, and G.W. Bryant, Phys. Rev. B 55, 10878 (1997).

[14] J.E.G.J. Wijnhoven, L. Bechger, and W.L. Vos, Chem. Mater. 13, 4486 (2001).

[15] M.L.M. Balistreri, J.P. Kortenk, L. Kuipers, and N.F. van Hulst, Appl. Phys. Lett. 77, 4092 (2000).

[16] B. Hecht, H. Bielefeldt, Y. Inoue, and D.W. Pohl, J. Appl. Phys. 81, 2492 (1997).

[17] R.W. James, The Optical Principles of the Diffraction of $X$-rays (Bell, London, 1954).

[18] R. Carminati, and J.J. Sáenz, Phys. Rev. Lett. 84, 5156 (2000).

[19] G. Colas des Francs, C. Girard, J.-C. Weeber, C. Chicane, T. David, A. Dereux, and D. Peyrade, Phys. Rev. Lett. 86, 4950 (2001).

[20] J.S. Kole, M. T. Figge, and H. De Raedt, Phys. Rev. E 64, 066705 (2001). 